\title{
La manipulation des espèces animales. Réflexion anthropologique sur la qualification du sauvage
}

Manipulating animal species. An anthropological reflexion on qualifying what is savage

\section{Colette Mechin}

\section{OpenEdition}

Journals

\section{Édition électronique}

URL : http://journals.openedition.org/economierurale/3399

DOI : 10.4000/economierurale.3399

ISSN : 2105-2581

\section{Éditeur}

Société Française d'Économie Rurale (SFER)

\section{Édition imprimée}

Date de publication : 1 mars 2012

Pagination : 143-151

ISSN : 0013-0559

Référence électronique

Colette Mechin, « La manipulation des espèces animales. Réflexion anthropologique sur la qualification du sauvage », Économie rurale [En ligne], 327-328 | janvier-mars 2012, mis en ligne le 01 mars 2014, consulté le 02 mai 2019. URL : http://journals.openedition.org/economierurale/3399 ; DOI : 10.4000/economierurale.3399 


\title{
La manipulation des espèces animales Réflexion anthropologique sur la qualification du sauvage
}

\author{
Colette MÉCHIN • Chercheur CNRS, Université de Strasbourg
}

\section{État des lieux}

$\mathbf{P}$ our renforcer des populations d'animaux sauvages menacées d'extinction, il faut disposer de sources d'approvisionnement en individus vivants. Soit on opère par capture en des lieux «d'abondance » pour placer sur des sites considérés en déficit, soit on élève in situ ou ex situ un nombre variable d'individus qui seront installés à un moment donné, dans un espace donné. À partir de récits concernant la réintroduction de différentes espèces, il s'agit de revisiter les scenarii des opérations de capture/relâcher (ou élevage/lâcher) et des situations qu'elles induisent et de permettre une réflexion concernant le statut de ces espèces à réintroduire (ou dont on veut « renforcer » la population) avec une attention particulière pour le cas du hamster commun (Cricetus cricetus L.) en Alsace, au sujet duquel je travaille depuis une dizaine d'années (Méchin 2005, 2007, 2011) ${ }^{1}$.

Concernant cette espèce protégée, il faut avoir en mémoire : i) que l'animal a longtemps été considéré comme un indésirable et détruit jusque dans les années 1990 ; ii) que la France a obligation d'empêcher la régression patente des populations sous peine d'une condamnation par la Cour de Justice européenne ${ }^{2}$ (accompagnée d'une astreinte financière). Dans cette perspective, le renforcement des populations a été jugé indispensable. Les autorités en charge du dossier ont dû «bricoler » des procédures d'élevage et de relâcher. Ces procé-

1. Un contrat FRB - LU (2010-2011) a été attribué à l'université de Strasbourg sur le thème « La biodiversité dans les plaines loessiques d'Alsace » pour poursuivre ce travail.

2. Mise en demeure de la Commission européenne en octobre 2007. dures font l'objet d'âpres critiques de la part de certaines associations de protection de la nature. De ce fait l'information est verrouillée ou édulcorée. Dans l'impossibilité de mener une enquête de type ethnographique classique, j'ai dû emprunter des « chemins de traverse » pour rencontrer un certain nombre d'acteurs et me faire une opinion de la situation. Cependant, nonobstant les particularités du dossier hamster, c'est à un niveau plus général que prétend se situer cet article pour faire ressortir, à travers des protocoles de déplacement, d'élevage, et de contrôle de populations protégées de la faune sauvage, une question ontologique primordiale (aux deux sens du terme) des conséquences tant au niveau concret que symbolique de ces manipulations. Elle concerne à la fois la qualification des espèces concernées et celle des usages des espaces ruraux, même si ce deuxième sujet ne sera pas abordé en profondeur'.

\section{Capturer et transporter}

Il existe un protocole de mesures établi par la Convention internationale sur le commerce des espèces menacées d'extinction (dites CITES ou Convention de Washington) pour permettre de déplacer des animaux d'un endroit à un autre, voire d'un pays à un autre. Ces actions mettent en cause plusieurs ministères puisque, en France, l'article L. 236-1 du Code rural prévoit, pour l'introduction sur le territoire français d'animaux apportés de l'étranger, d'être soumis à des exigences fixées par le ministre de l'Agriculture. «Celles-ci doivent permettre de s'assurer que seuls sont introduits des animaux en bonne santé générale

3. Article en préparation. 
et n'exprimant aucun signe clinique de maladie, en particulier de rage. ${ }^{4}$

Ainsi, concernant la situation de l'ours, conformément aux préconisations formulées, "le risque sanitaire associé aux translocations d'ours slovènes et croates a été examiné par les vétérinaires de l'ONCFS ${ }^{5}$ au cours d'une étude. Celle-ci a bénéficié de l'appui scientifique et technique de l'agence française de sécurité sanitaire des aliments (AFSSA) ».

Ensuite, toujours à propos d'ours slovène réintroduit dans les Pyrénées, il a fallu une « autorisation de transport en vue du relâcher dans la nature de spécimens d'espèces animales protégées, délivrée par le ministère de l'Écologie et du Développement durable après avis du Conseil national de la protection de la nature ${ }^{6}{ }^{6}$

Les protocoles de capture et de transport sont très précis. Dans un document consultable sur le site de l'ONCFS il est expliqué : «Les animaux sont capturés à l'aide de piège de type Aldrich ou par télé-anesthésie à partir d'affûts disposés sur des sites où de la nourriture est déposée pour les attirer. La manipulation de l'animal demande selon les cas entre 40 et 120 minutes. Chaque ours capturé est équipé d'un collier émetteur VHF et parfois muni d'une marque auriculaire. Pour les trois projets de restauration de l'ours brun [en Autriche, en France et en Italie], le transport des animaux s'est fait par la route dans une cage installée dans un camion » (Quenette et al., 2006).

D'autres exemples d'espèces réintroduites peuvent être convoqués ${ }^{7}$ à l'exemple du

4. Pour la bibliographie concernant les protocoles de translocations des espèces animales on consultera Mauz (2006).

5. Office national de la chasse et de la faune sauvage.

6. Site ministère de l'Écologie, de l'Énergie, du Développement durable et de l'Aménagement du territoire : www.ours.ecologie.gouv.fr

7. On n’évoquera pas ici «les récits de (ré) introductions pleins d'épisodes imprévus [...] parfois franchement rocambolesques » dont fait mention Mauz (2006). castor qui a pu recoloniser progressivement le bassin rhodanien. Il a servi de source d'approvisionnement pour la réintroduction dans d'autres régions de France (sa protection a été étendue en 1968 à tout le territoire national). Il avait été réintroduit de longue date dans d'autres pays d'Europe : d'abord dans les pays scandinaves (vers les années 1930), puis en France et en Suisse (à partir des années 1960), puis en Pologne (à compter des années 1970), et plus récemment aux Pays-Bas.

Rouland (1991) rappelle que «les techniques de capture, de contention et de conservation de courte durée des castors sont bien maîtrisées. Depuis 1981, les agents de l'Office national de la chasse ont assuré toutes les captures nécessaires aux besoins de réintroduction, sous couvert des autorisations correspondantes du ministère de l'Environnement ». Il explique : "Pour des raisons relatives à la biologie de l'espèce et à la technique de capture, les prélèvements - et donc les lâchers - sont réalisés d'octobre à début avril sur des sites où les castors posent des problèmes. Les animaux peuvent rester trois jours en captivité, au calme et à l'abri, le temps d'opérer un regroupement familial. Dès la fin de l'opération de capture, les individus appartenant à une même famille sont transportés et lâchés simultanément sur le site choisi au préalable. Il n'y a donc pas lieu d'installer un parc de prélâcher ni de prévoir des gîtes artificiels. À l'examen des résultats des opérations menées à ce jour, nous estimons à trois familles, soit 10 à 15 individus, l'effectif minimum pour réussir une réintroduction en masse. Les différentes familles sont lâchées sur des sites distants de 5 à 10 km afin d'éviter un contact immédiat entre des individus territoriaux. La mortalité constatée est faible sur ces 49 animaux: aucun cas à la capture, 1 cas entre la capture et le lâcher (pancréatite, stress?), 3 cas dans les semaines qui ont suivi le lâcher : 1 écrasement par véhicule (Moselle), 1 chute dans un puits (Dourbie), 1 cas de cause inconnue (Drac). À titre de comparaison, en Pologne, 
sur 168 castors réintroduits sur le bassin de la Vistule, $5 \%$ sont morts à la capture et $14 \%$ dans l'année qui a suivi le lâcher. »

Dans le cas du hamster commun, espèce qui ne subsiste plus sur le territoire français qu'en Alsace, les protocoles de capture et de déplacement ont été progressivement mis en place par l'ONCFS à partir de $1998^{8}$. Ils nécessitent les mêmes autorisations que pour les espèces plus volumineuses. Ils ont conduit soit au déplacement des individus sur des parcelles d'agriculteurs « conventionnés » soit à leur placement dans un site d'élevage (dans un parc à Hunawihr, sur un site d'élevage à Elsenheim, dans une moindre mesure au zoo de Mulhouse, et d'une autre manière - on y reviendra - dans un laboratoire CNRS).

\section{Élever et installer}

Lorsque les circonstances l'exigent, la réintroduction d'une espèce menacée passe par l'élevage. Concernant les espèces sauvages dont la valeur patrimoniale est reconnue ${ }^{9}$, l'élevage nécessite un personnel qualifié pour procéder non pas tant à la survie d'un individu donné (un ours blanc, un tigre, un éléphant dans un zoo par exemple) qu'à l'accession à la maîtrise de l'ensemble des processus physiologiques et biologiques (y compris la reproduction) de l'espèce en question. La définition de ce métier est, à y bien regarder, celle exigée des techniciens qui élèvent des animaux de laboratoire, même si la finalité en est sensiblement différente $^{10}$. Dans le cas du hamster commun, c'est d'ailleurs un laboratoire de neurosciences du CNRS à Strasbourg qui, par la force des circonstances, se trouve au centre de cette stratégie. Ce laboratoire, grand

8. Le plan de conservation date de 1995 (Losinger et al., 2006).

9. Espèces protégées par la Convention de Berne. 10. Dans les associations autorisées à faire de l'élevage (cas du hamster en Alsace) un certificat de capacité est requis. spécialiste des cycles biologiques et de ses troubles, a longtemps travaillé avec des hamsters prélevés dans l'espace agricole alsacien. Lorsque l'espèce a été protégée et qu'il a été interdit de poursuivre l'approvisionnement du laboratoire par prélèvement direct, l'équipe s'est tournée vers l'élevage de cette espèce (mais on y élève aussi des hamsters dorés, sibériens...). En 2008, ce laboratoire, en perspective du recrutement d'un technicien (on le désigne « animalier ») détaille ainsi les activités : «Assurer l'entretien des animaux et leur hébergement (renouvellement des litières, distribution de la nourriture et de la boisson, animaux en cages ouvertes ou portoirs ventilés). Réaliser des accouplements et sevrages. Surveiller et respecter l'environnement des animaux : air (température, humidité, filtration), lumière (horloge), suppression des nuisances (bruits, intrusion). Appliquer les réglementations relatives à une unité d'élevage (hygiène, sécurité) et les bonnes pratiques de laboratoire relatives à l'expérimentation animale. Savoir rendre compte de tout dysfonctionnement. Entretenir les locaux et le matériel destiné à l'unité d'élevage. Gérer l'évacuation des déchets en fonction de leur catégorie et des risques, dans le respect des règles d'hygiène et de sécurité de l'unité. Participer à la tenue des registres et fichiers. Participer en rotation aux astreintes de week-end et jours fériés ${ }^{11}$.

Dans une interview donnée en 2007, le directeur de ce laboratoire décrit ainsi les installations : «Il s'agit d'un équipement spécifique conçu pour étudier les rythmes biologiques, c'est-à-dire les mécanismes cérébraux que possèdent les animaux pour percevoir et s'adapter aux changements de l'environnement. Le bâtiment, unique en Europe, contient des installations qui permettent de reproduire les changements du milieu de manière contrôlée, essentiel-

11. Site Internet : http://web-rh.dsi.cnrs.fr au 10/06/2008. 
lement la photopériode (l'alternance jour/nuit). On peut donc varier la longueur du jour et de la nuit, la température (utile pour étudier l'hibernation), mimer l'aube et le crépuscule, contrôler l'humidité. Bref, dans ce bâtiment, nous pouvons avoir la maîtrise de tous les facteurs physiques $d u$ milieu $\gg .^{12}$

Le cahier des charges concernant l'élevage des animaux utiles à l'expérimentation n'est pas particulier à ce laboratoire. Le responsable de l'animalerie du campus CNRS de Gif-sur-Yvette qui élève des rongeurs, des poissons, des lapins pour les différents laboratoires du campus détaille les différentes activités qu'il doit réaliser : « Apporter tout le soin nécessaire aux animaux (change, nourriture, boisson) ; être attentif au bien-être des animaux; fournir une assistance aux chercheurs lors des protocoles d'expérimentation animale (préparer les animaux, réaliser des prélèvements de sang, effectuer des injections...); produire des anticorps pour les laboratoires $\gg .^{13}$

La mise en œuvre de mesures permettant la sauvegarde du hamster commun ayant été jugée insuffisante par la Commission européenne ( $c f$. supra), le « renforcement des populations » par des lâchers d'animaux d'élevage en grand nombre a été retenu. Par un étrange retournement de la situation qui ne peut pas laisser indifférent, c'est ce laboratoire CNRS, longtemps critiqué pour ses prélèvements d'animaux d'expérimentation qui, devenu maître en la matière pour reproduire l'espèce, est maintenant sollicité pour alimenter les lieux d'élevage ${ }^{14}$. L'information, si elle n'est pas tenue secrète, est cependant occultée par tous les acteurs du dossier. Les « gens ordinaires » (les agriculteurs particulièrement)

12. http://www.journaldunet.com/science/biologie/ interviews07/pevet.shtml.

13. In Préventions Infos, CNRS, septembre 2008. 14. Pas de chiffres communiqués. En 1999 le laboratoire disposait « de plus de 300 animaux » (Plan de conservation, novembre 1999). n'ont pas connaissance de cette clause puisque les sites d'élevage sont sous la responsabilité d'une association (Sauvegarde Faune Sauvage) et qu'on ne mentionne pas le rôle décisif en la matière de «naisseur » de ce laboratoire. Tout se passe comme s'il était préférable, dans une perspective pragmatique d'efficacité, d'ignorer ce paradoxe.

Une fois mené à bien l'élevage proprement dit, vient la phase d'installation des animaux. Il faut d'abord rappeler que le placement de ces animaux sauvages d'un nouveau type, sur un terrain approprié, qu'ils soient changés d'un lieu à un autre ou qu'ils proviennent d'un élevage, nécessite, outre un savoir-faire élémentaire, une autorisation administrative extrêmement contraignante. Vient ensuite le protocole d'installation proprement dite :

- À propos des lynx il est expliqué ${ }^{15}$ : «Avant d'être relâchés dans leur nouveau domaine [les lynx] sont vaccinés, tatoués et munis d'un collier émetteur sous anesthésie, puis ils subissent un traitement fortifiant de vitamines et d'huile de foie de morue. » Ensuite, « un suivi journalier permet de localiser les lynx et a ainsi permis une meilleure compréhension de cet animal et de ses mours. »

- Concernant les ours, on lit sur un site internet $^{16}$ : «L'ours vivant en liberté en Slovénie ou en Croatie est un animal robuste et peu sujet aux maladies. L'analyse effectuée montre clairement que le risque sanitaire lié à la translocation est faible. De plus la mise en ouvre de mesures simples suffit à rendre ce risque négligeable : les principales mesures préventives ou curatives préconisées avant la réintroduction consistent en des traitements antiparasitaires externes et internes [pas de vaccinations préconisées]. »

15. www.lelynx.org/Reintroduction 16. www.oncfs.gouv.fr 
Mais une surveillance régulière sera effectuée pendant une période de six mois après le lâcher dans le milieu naturel. Durant cette période : «Un rapport relatif à l'état de santé de l'animal établi par un vétérinaire, soit à partir d'une observation directe, soit par analyse [fèces, urine] ou tout autre méthode appropriée sera transmis au directeur départemental des services vétérinaires du lieu d'accueil à la fin de chaque mois ; tout comportement suspect laissant supposer l'apparition d'une pathologie, et en particulier la rage, fera immédiatement l'objet d'une information au directeur départemental des services vétérinaires du lieu d'accueil. »

- En Alsace, à propos du hamster, où on parle « d'opération de renforcement » et non de réintroduction, l'installation des hamsters sur un site se modifie d'année en année, en fonction des acquis de l'expérience. «[...] les premières analyses menées en 2005 montraient que les individus s'échappaient relativement rapidement quelle que soit la technique utilisée [faux terriers ou cages]. Leur premier réflexe était de tester l'imperméabilité de l'enclos et de creuser au niveau des angles pour s'enfuir. » En 2006 est choisie une technique de faux terrier fermé par des tuiles qui a l'avantage «d'être plus discret dans la végétation et donc moins repérable par les prédateurs [rapaces et renards postés près des cages]. »

- Au sujet du lâcher de 50 hamsters (22 mâles et 28 femelles) qui eut lieu le 31 mai 2006, le rapport ONCFS explique : «Les animaux ont été transférés de leur cage de transport jusqu'aux ébauches de terriers à l'aide du filet servant à leur manipulation en captivité par la responsable de l'élevage. [...] Toutes les demi-heures et jusqu'à la tombée de la nuit, un passage à proximité des ébauches de terriers a été effectué pour vérifier si l'animal était encore présent. [...] Le $1^{\text {er }}$ juin, une nouvelle vérification de l'occupation a été effectuée. Seuls deux hamsters communs [...] étaient encore présents et n'avaient pas cherché à sortir. »

Que ce soit pour l'ours, le lynx ou le hamster, on est frappé par l'extrême « encadrement » de ces différentes populations d'animaux par un personnel nombreux (souvent bénévole) et omniprésent sur les sites. Cette présence attentive et interventionniste pose, d'une certaine façon, le problème évoqué en son temps par Micoud (1993), dans un article fondateur sur la catégorisation du sauvage.

\section{Le sauvage requalifie}

Les différentes étapes de l'installation d'une faune sauvage disparue ou en voie d'extinction obligent à revenir sur l'impact de ces manipulations (au sens où l'implantation ne se fait pas naturellement) au niveau de ce que l'on désignera par la « symbolique » ou « le système des représentations ». Raison du Cleuziou (2008) ${ }^{17}$ propose de désigner comme « requalification scientifique des objets naturels » ce moment où certains animaux deviennent l'enjeu potentiel de conflits sociétaux (dans le cas qu'il étudie, il s'agit des tritons des mares de la baie de Somme). Mais lorsqu'il s'agit de réintroduction, ce n'est plus seulement la « qualification » qui est concernée mais le principe même de la notion de sauvage qui est affecté.

Le contrôle par marquage/tatouage (identification), localisation par transpondeur ou non, capture-recapture, vérifications sanitaires, voire apport de nourriture ${ }^{18}$, oblige à reconsidérer le statut des espèces concernées dans une classe nouvelle, peut-être celle définie par Latour comme celle des hybrides : «Tant que la nature était lointaine et dominée elle ressemblait encore vaguement au pôle constitutionnel de la

17. In page 380.

18. Concernant le hamster commun le projet réapparaît régulièrement de ce «suivi » à des fins de collecte des données. 
tradition. Elle semblait en réserve, transcendante, inépuisable, lointaine. Mais où classer le trou de l'ozone, le réchauffement global de la planète ? Où sont les hybrides? Sont-ils humains? Humains puisque c'est notre auvre. Sont-ils naturels? Naturels puisqu'ils ne sont pas notre fait. » (Latour, 1997) ${ }^{19}$.

Les processus de capture, d'élevage, de suivi de ces espèces réintroduites rendent bien compte de cette transgression d'une frontière : dans la littérature concernant ce sujet, apparaît l'idée que certains animaux élevés en captivité ont perdu « la peur de l'homme ${ }^{20}$, qu'ils ne savent pas se débrouiller par eux-mêmes (pour chercher leur nourriture) ou, dans le cas du hamster, sont sans défense face aux prédateurs (avis d'un responsable néerlandais rencontré en 2005, site de Sibbe). Soit dit en passant, on assiste, dans le même temps, à un « ensauvagement» des bêtes de troupeau comme le montre Luxereau (1995), à propos des vaches des Pyrénées ariégeoises.

Dans le cas particulier du hamster en Alsace, le « renforcement » des populations ayant échoué par simple déplacement, l'élevage s'est développé, à l'initiative de l'association Sauvegarde Faune Sauvage (d'abord au zoo de Mulhouse, puis au Centre de réintroduction des cigognes et des loutres de Hunawihr et, enfin, sur un site prêté par un agriculteur à Elsenheim). La nécessité de mettre à contribution un laboratoire CNRS, qui utilise encore aujourd'hui le hamster commun comme animal de laboratoire, pour permettre une extension de l'élevage (et la survie de l'espèce) repose, on l'a vu, sur un paradoxe. De ce fait, on est passé d'une forme de «production » à un «élevage » (avec changement de perspective puisque le matériel vivant à finalité expérimentale devient un « en soi » vivant qui doit perdurer). Dans ce contexte, on demande au(x) zootechnicien(s) qui

19. In page 97.

20. Sur cette notion de « distance », $c f$. Mauz (2002). contrôle(nt) bien la reproduction du hamster en milieu artificiel (laboratoire) de réaliser la même chose à destination du milieu naturel ${ }^{21}$.

Aussi pragmatique que soit le procédé, on ne peut qu'être frappé par la proximité (promiscuité ?) des techniques d'élevage mises en place avec celles des élevages à finalité commerciale. Avec, dans un but d'efficacité évident, une répartition des activités et une spécialisation des acteurs : les techniciens du laboratoire CNRS sont « naisseurs », les membres de l'association Sauvegarde Faune Sauvage sont « éleveurs » (puis réintroducteurs). La division du travail est, qu'on le veuille ou non, construite selon un modèle bien connu des élevages intensifs. On retrouve les impératifs d'un élevage rationnel, fondés sur une bonne connaissance des mécanismes biologiques, une maîtrise de tous les paramètres concernant aussi bien l'alimentation, le confort des cages, les problèmes sanitaires que la température, la luminosité etc. (Sauveur, 1988). On est certes loin d'atteindre une production « industrielle », même si le souci des administrations en charge du dossier est d'augmenter le nombre d'individus à relâcher. D'après les chiffres récupérés ici ou là, on est passé d'une vingtaine d'individus en 2004 (Losinger et Petiteau, 2005) à 75 en $2006^{22}$, à 170 en $2009^{23}$.

La réflexion sur le statut de cet animal «fabriqué »s'impose. À propos des élevages intensifs, Sigault, (1991) s'interroge sur cette « matière première » d'un nouveau genre et Salmona (1986) parle, elle, du « traitement industriel du vivant », mais que dire quand l'espèce concernée

21. Compte tenu de la difficulté déjà soulignée à obtenir des informations, et le souci de pouvoir continuer à travailler sur ce sujet politiquement « sensible », je n'ai pas essayé de rencontrer l'animalier de ce laboratoire.

22. In Bilan des mises en œuvre des activités techniques en 2006, ONCFS.

23. $C f$. site internet de Sauvegarde Faune Sauvage, juin 2009. 
n'est pas domestique ? Quand, aux préoccupations de performance propres aux élevages intensifs, s'ajoutent, contradictoirement, une remise en situation «naturelle » des individus ainsi produits (avec les questions de leur capacité à se nourrir, à se défendre des prédateurs, bref à survivre à ces nouvelles conditions d'existence)?

On remarquera que cette situation se développe de manière concomitante à une redéfinition de l'espace rural comme environnement à manager et à protéger ${ }^{24}$, avec désignation d'espaces particuliers définis selon des critères nouveaux d'après Jollivet (1997). En Alsace ce sont des « secteurs noyaux », des « aires vitales » et des « zones d'actions prioritaires » qui redécoupent l'espace au gré des impératifs des décideurs. Cette « sanctuarisation » de la nature fait écrire à Raison du Cleuziou (2008) : «Avec les politiques européennes de protection de l'environnement, un zonage administratif et normatif requalifie l'espace en fonction d'une finalité de durabilité qui induit un double changement d'échelle : celui $d u$ temps long qui s'intéresse à un devenir à garantir, celui d'un espace à considérer sous l'angle transeuropéen voire planétaire [espèce menacée dans sa spécificité locale]. » Et, plus loin : «L'espace rural est découpé en territoires définis par des critères scientifiques (les Zones naturelles d'intérêt écologique faunistique et floristique, ZNIEFF ; les Zones spéciales de conservation, ZSC ; les Zones de protection spéciale, ZPS). »

Cette « construction savante de la nature » (Raison du Cleuziou, 2007) est définie par de nouveaux acteurs appelés à régir le territoire en définissant non seulement la nature mais la place des animaux « considérés comme légitimes, indifférents ou nuisibles ». Dans ce contexte, les agriculteurs de la plaine alsacienne se sentent dépouillés symboliquement de leur légitimité puisque

24. Micoud et Bobbé proposent la notion de « campagne écologisée » (2006). les « zonages » sont décidés (modifiés, élargis ${ }^{25}$ ) par des experts. Pour permettre le « retour» du hamster en Alsace, il aura fallu modifier les conditions socio-économiques locales. J'ai présenté ailleurs ${ }^{26}{ }^{1}$ 'animosité profonde développée par nombre d'agriculteurs alsaciens concernant cette « vermine » qu' on avait le droit de détruire par tous les moyens possibles jusque dans les années 1990.

Les sollicitations faites aux agriculteurs pour faire une place à l'animal en adaptant et modifiant leurs pratiques culturales deviennent pressantes devant les menaces de sanction européennes Il a fallu, dès 2000, indemniser les dégâts constatés dans certaines cultures (les choux, mais pas les céréales), démarcher les agriculteurs pour signer des conventions avec ceux qui accepteraient de :

1) ne pas détruire les hamsters installés sur leurs terres,

2) modifier leurs pratiques culturales permettant la survie de l'animal.

Le cahier des charges tel qu'il est proposé depuis 2008 offre différentes aides financières pour ceux qui mettent en place de la luzerne dans leur rotation de culture (529€ par ha en 2010) ou des céréales d'hiver (205 € par ha) avec obligation de conserver une bande non fauchée au moment de la récolte, de ne pas travailler le sol avant le 15 septembre, de ne pas labourer à plus de $30 \mathrm{~cm}$ de profondeur, de ne pas utiliser des insecticides et rodenticides sur ces parcelles.

Même si l'obligation de faire une place au hamster en Alsace n'est évidemment pas la raison de l'interrogation du système agricole actuel, elle participe cependant à cette logique de remise en cause du modèle d'une

25. «Vous élaborerez d'ici le mois de septembre une proposition de modification des périmètres des ZAP, en particulier pour couvrir les secteurs situés à proximité des ZAP actuelles... » (Courrier du 28 août 2009 du secrétariat d'État chargé de l'Écologie). 26. $C f$. Méchin, 2005, 2007, 2011. 
agriculture intensive. La gageure est, de surcroît, de faire accepter un animal qui n'a jamais cessé d'être considéré comme un « nuisible » et qu'on « élève » maintenant au grand scandale de la population agricole. Et le vivant sauvage qui évolue dans cette nature d'un nouveau type «n'est plus le sauvage de [nos] rêves. Observé, compté, soigné, il est un sauvage "naturalisé vivant» ou en "liberté surveillé » et dont, le droit à l'existence étant reconnu par tous, il convient de s'entendre entre tous sur la manière de gérer publiquement les modalités de sa régulation » (Micoud et Bobbé, 2006). Reconnu par tous ? En Alsace le consensus n'est toujours pas évident... 


\section{RÉFÉRENCES BIBLIOGRAPHIQUES}

Jollivet M. (1997). Pour un rural postindustriel. Paris, L'Harmattan. 230 p.

Latour B. (1997). Nous n'avons jamais été modernes. Essai d'anthropologie symétrique. Paris, La Découverte, 198 p.

Losinger I., Petiteau M. (2005). First results of the reinforcement program monitoring of Common Hamster population in Alsace. In "Actes du colloque international sur le Grand Hamster" (Cricetus cricetus L.), Strasbourg, ONCFS.

Losinger I., Wencel M.-C., Migot P. (2006). Réflexions autour de la gestion d'une espèce animale dans un écosystème agricole : le cas du grand hamster, Natures, Sciences, Sociétés, ${ }^{\circ}$ 14. p. S63-S66.

Luxereau A. (1995). Les vaches d'avant étaient méchantes, celles de maintenant sont des bâtardes. Changement de races en Couserans (Pyrénées ariégeoises). In Lizet B., Ravis Giordani G. (éd.) « Des bêtes et des hommes », Paris, CTHS, p. 93-105.

Mauz I. (2002). Les conceptions de la juste place des animaux dans les Alpes françaises. Espaces et Sociétés, p. 129-145.

Mauz I. (2006). Introductions, réintroductions : des convergences par delà les différences. Natures, Sciences, Sociétés, vol. 14 suppl., p. S3-S10.

Méchin C. (2005). For an ethnological approach of the Common Hamster in Europe. In «Actes du colloque international sur le Grand Hamster » (Cricetus cricetus L.). Strasbourg, ONCFS.

Méchin C. (2007). La gestion de l'espace rural et périurbain et les enjeux de sauvetage d'une espèce protégée : la situation du hamster commun (Cricetus cricetus L.) en Alsace. Actes du colloque «Les mondes ruraux à l'épreuve des sciences sociales », Dijon, INRA, p. 365-377.
Méchin C. (2011). Une espèce protégée qui dérange : le hamster commun (Cricetus cricetus L.) en Alsace. Anthropozoologica, vol. 46, $\mathrm{n}^{\circ}$ 1, p. 127-139

Micoud A. (1993). Vers un nouvel animal sauvage : le sauvage «naturalisé vivant ». Natures, Sciences, Sociétés, vol. 1, n 3 , p. 202-210.

Micoud A., Bobbé S. (2006). Une gestion durable des espèces animales est-elle possible avec des catégories naturalisées ? Natures, Sciences, Sociétés, vol. 14, p. S32-S35.

Quenette P.-Y., Rauer G., Huber D., Kazensky P., Knauer F., Mustoni A, Palazon S., Zibordi F. (2006). Comparaison du comportement spatial d'ours bruns réintroduits et non réintroduits en Europe. ONCFS, téléchargeable sur www.oncfs.gouv.fr

Raison du Cleuziou Y. (2007). Le destin politique des bêtes : l'embrigadement des animaux dans des constructions sociales de la nature concurrentes. Actes du colloque «Les mondes ruraux à l'épreuve des sciences sociales ». Dijon, INRA, p. 378-390.

Raison du Cleuziou Y. (2008). De la résistance à la subversion. Les chasseurs de la baie de Somme et le développement durable. Études rurales, ${ }^{\circ} 181$, p. 133148.

Rouland P. (1991). La réintroduction du castor en France. Le courrier de l'environnement, $\mathrm{n}^{\circ} 14$, p. 35-42.

Salmona M. (1986). Les femmes et l'élevage. Ethnozootechnie, ${ }^{\circ} 38$, p. 91-106.

Sauveur B. (1988). Reproduction des volailles et production d'œufs. Paris, INRA, $355 \mathrm{p}$.

Sigault F. (1991). L'animal, machine ou personne? Ethnozootechnie, n ${ }^{\circ}$ 46, p. 5-12. 